\title{
Salud laboral y bienestar. Incorporación de modelos positivos a la comprensión y prevención de los riesgos psicosociales del trabajo
}

\author{
Occupational health and well-being: incorporation of positive models to the un- \\ derstanding and the prevention of psychosocial risk
}

\section{Eva Garrosa Hernández, Isabel Carmona Cobo}

\section{Facultad de Psicología, Universidad Autónoma de Madrid.}

\author{
Correspondencia: \\ Eva Garrosa Hernández \\ Facultad de Psicología \\ Universidad Autónoma de Madrid \\ C/ Ivan Pavlov, 6 \\ Ciudad Universitaria de Cantoblanco \\ 28049 Madrid. España. \\ Tfno.: + 34914973384 \\ E-mail: eva.garrosa@uam.es
}

Resumen

Las variables de personalidad positiva del trabajador tienen una función decisiva en el desarrollo de los recursos personales que ayudan a los trabajadores a orientarse a la salud y el bienestar, y al óptimo desarrollo laboral. Desde esta perspectiva, se trata de exponer algunas de las teorías más representativas que intentan ofrecer una aproximación y explicación de los mecanismos implicados e intervinientes.

Potencialmente, se pretende explorar y ofrecer un recorrido sobre cómo los aspectos positivos organizacionales y personales pueden tener una vinculación concluyente con la productividad, creatividad, y calidad del trabajo realizado, esto es, con los resultados organizacionales. Desde esta configuración, se va más allá de la clásica línea de prevención de los riesgos laborales y se intenta comprender cuáles son los factores organizacionales, laborales y personales que promueven la salud y el bienestar en las organizaciones, tanto para los trabajadores como para los usuarios del servicio prestado. Por último, se muestran algunos ejemplos que desde estas perspectivas pueden contribuir en la realización de buenas prácticas laborales.

Palabras clave: bienestar, emociones positivas, engagement, personalidad positiva, recursos personales, salud laboral.

\begin{abstract}
Workers' positive personality variables have a crucial role in the development of personal resources which help workers to provide guidance to health and well-being, and optimum employment development. From this perspective we have exposed some of the most representative theories that try to offer an approximation and explanation of the mechanisms involved.

Potentially, we aim to explore and offer a general view on how organizational and personal strengths may have a conclusive linkage with productivity, creativity, and quality of work, that is, with the organizational results. From this configuration, we go beyond the classic line of occupational risks prevention trying to understand the organizational, work and personal factors that promote health and well-being in organizations, both for workers and the users of the service provided. As a final point, some examples are discussed which can help in the realization of good labour practices.
\end{abstract}

Keywords: well-being, positive emotion, engagement, positive personality, personal resources, occupational bealth. 


\section{INTRODUCCIÓN}

La salud laboral desde la perspectiva de la psicología, también ha sido influida por la emergencia de la psicología positiva ${ }^{1}$ en el sentido de tratar de buscar y comprender cuáles son las variables personales y recursos que conseguirían una mayor adaptación, salud y bienestar para los trabajadores. Las variables de personalidad positiva del trabajador activarían los recursos personales que ayudan a los trabajadores a orientarse a la salud y el bienestar, y al óptimo desarrollo laboral.

Al mismo tiempo, también se ha producido una aproximación con los modelos de la Psicología Organizacional Positiva preocupada por conocer en qué medida los aspectos positivos organizacionales pueden tener una implicación decisiva a nivel de productividad, creatividad, y calidad del trabajo realizado, esto es, en los resultados organizacionales. De buena parte de la combinación de estas perspectivas surge lo que a nivel internacional se ha denominado como Positive Occupational Health Psychology (POHP) ${ }^{2}$.

Desde esta configuración, se va más allá de la clásica línea de prevención de los riesgos laborales (p.ej., estrés y burnout) y se trata también de conocer cuáles son los factores organizacionales, laborales y personales que promueven la salud y el bienestar en las organizaciones, tanto para los trabajadores como para los usuarios del servicio prestado.

Una concepción errónea de este enfoque sería centrarse exclusivamente en fomentar o desarrollar las variables del trabajador que le pueden ayudar a nivel de resistencia en las condiciones adversas laborales, sin realizar cambios organizacionales necesarios. Desde esta perspectiva se pretende desarrollar contextos laborales positivos donde el respeto y las conductas cívicas sean la norma, así como generar posibilidades de desarrollo profesional y entornos amigables donde el trabajador pueda conciliar en mayor medida su vida laboral y personal. En definitiva, un entorno laboral saludable.

Al mismo tiempo, las variables personales positivas ayudan a favorecer este entono saludable laboral y desarrollar soluciones creativas frente a los problemas laborales. En el caso de que el trabajador esté sufriendo un entorno tóxico de trabajo, estas variables personales le ayudarán a afrontar mejor la situación y le pueden ayudar a resolver el problema desde una perspectiva realista, con la valoración de si conveniente seguir en este entorno o si se pueden conseguir cambios significativos de otro modo.

\section{EMOCIONES POSITIVAS, SALUD Y BIENESTAR LABORAL}

Dentro de los modelos de emociones positivas, es el modelo de Barbara Fredrickson ${ }^{3}$ que propone con la teoría de ampliación y construcción de las emociones positivas, que contribuyen al crecimiento y el desarrollo de la persona, también tiene implicaciones en el medio laboral. Los contextos laborales donde las emociones negativas son frecuentes (p.ej., la ira o la ansiedad) limitan el repertorio pensamiento-acción del trabajador, y entre entras consecuencias, su creatividad y desarrollo eficaz. De manera opuesta, las emociones positivas amplían este repertorio, ofreciendo la oportunidad de desarrollar recursos personales duraderos, que a su vez, ofrecen la posibilidad de crecimiento personal mediante la creación de espirales positivas o adaptativas de emoción, cognición y acción, con el consecuente desempeño laboral sobresaliente. Por ejemplo, la alegría genera un clima laboral positivo, fortalece las redes sociales de apoyos laborales, el trabajo en equipo, la creatividad y la resolución creativa de los problemas laborales, la productividad y el bienestar del trabajador.

Las emociones que sienten los trabajadores son una parte muy importe del bienestar laboral, ya que son necesarias para fomentar la salud en las organizaciones, influyendo en el clima laboral positivo, en la calidad del trabajo que se realiza y en el servicio prestado. Las emociones positivas tienen una influencia importante sobre la motivación, la creatividad y el rendimiento en la tarea del trabajador ${ }^{4}$. 
Los profesionales se benefician de las emociones positivas, ya que éstas influirán de manera importante en la organización, generando un clima laboral positivo y contribuyendo en las relaciones interpersonales, generando entornos cívicos y amigables, donde la cooperación y el trabajo en equipo son productivos. Igualmente, este entorno permite cambios constructivos y mejora continua de las condiciones adversas a través por ejemplo, de programas psicoeducativos. Los trabajadores pueden diferenciar las condiciones laborales que no son óptimas para el bienestar, y este proceso de crítica constructiva posibilita un continuo cambio y mejoría en la dirección de la salud laboral y el bienestar.

Equivalentemente, es importante que el profesional sea sensible a las emociones de los clientes/usuarios y compañeros para manejar adecuadamente las situaciones de conflictos interpersonales y de regulación emocional, que se derivan de las situaciones cotidianas laborales, especialmente en las profesiones asistenciales. Estos elementos pueden prevenir también las situaciones de agresión laboral, ya que de esta manera se rompe la espiral de agresión que suele darse entre las conductas incívicas y que pueden llevar a situaciones de agresión continua y entorno laborales tóxicos 5 .

Por lo tanto, son importantes las investigaciones que han demostrado los efectos de las emociones positivas a nivel de prevención de riesgos laborales ${ }^{6}$, motivacional y de las acciones eficaces y la productividad asociada ${ }^{7}$, el aumento de la creatividad ${ }^{8,9}$, una mayor flexibilidad cognitiva y mayor apertura a las nuevas experiencias ${ }^{10}$, desarrollo de feedback constructivo y mayor pensamiento crítico ${ }^{11}$. A nivel interpersonal, las emociones positivas generan un sentimiento de mayor vinculación con los otros ${ }^{12}$, actitudes cívicas y empatía ${ }^{13}$ lo que sin duda contribuye a entornos laborales positivos y al bienestar del trabajador.

\section{ENGAGEMENT}

Otro de los modelos más influyentes en POHP para explicar el bienestar laboral, ha sido el modelo de demandas y recursos laborales ${ }^{14-18}$ que especifica cómo los trabajadores pueden orientarse y conseguir el bienestar laboral a través de la equilibrada interacción de las demandas y los recursos laborales. Las demandas laborales se refieren a los aspectos físicos y psicosociales de la organización que requieren un importante esfuerzo, y coste físico y psicológico (p. ej., emocional, cognitivo y conductual) por parte del trabajador ${ }^{19}$.

Los recursos laborales son aspectos del trabajo que permiten hacer frente a las demandas laborales y además facilitan el desarrollo personal y profesional, así como el aprendizaje de nuevos recursos y nuevas capacidades de perfeccionamiento y progreso ${ }^{20,6}$. Desde esta perspectiva, las demandas laborales y los recursos estarían negativamente relacionados, así, las demandas laborales como el estrés, las demandas emocionales de la interacción con pacientes movilizan recursos personales ${ }^{15,17}$. No obstante, los recursos personales no son una fuente inagotable y en este sentido la organización debe posibilitar recursos de apoyo al trabajador, por ejemplo, para que pueda llevar a cabo procesos de recuperación.

El modelo de demandas y recursos laborales (JD-R model) ${ }^{17}$ sugiere dos procesos psicológicos interrelacionados. Concretamente, las demandas del trabajo (p. ej., estrés de rol) suponen un importante agotamiento de los recursos emocionales del trabajador que puede extenuarlo y llevarlo a la situación de agotamiento emocional ${ }^{15,21}$. De manera contraria otros recursos personales orientan al trabajador a un proceso energético, un estado motivacional positivo, denominado engagement. Desde esta perspectiva los recursos organizacionales y personales están vinculados al engagement y los resultados positivos en las organizaciones, como el bienestar laboral ${ }^{18}$

Así, dentro de los modelos de bienestar laboral, donde los trabajadores se muestran conectados psicológicamente con su trabajo, surge el modelo de engagement, que comprende una visión contemporánea de las organizaciones donde los trabajadores se encuentran vinculados a su rol profesional, y que genera conductas proactivas y de 
compromiso con la realización del trabajo. Los trabajadores con engagement manifiestan energía y dedicación en su trabajo ${ }^{22}$. Las investigaciones han demostrado que engagement está relacionado con una buena realización laboral ${ }^{23,24}$, satisfacción de los clientes ${ }^{25}$, beneficios económicos para las empresas ${ }^{26}$ y bienestar laboral ${ }^{6}$.

Engagement con frecuencia se ha definido como un sentimiento positivo relacionado con el trabajo, que implica un estado de bienestar psicológico caracterizada por vigor, dedicación y absorción ${ }^{27,28}$. En definitiva la definición recoge el sentimiento positivo de estimulación y energía que genera el trabajo, que tiene un significado y que genera una completa concentración. Desde este punto de vista, se ha observado que los trabajadores con engagement muestran una mayor energía y autoeficacia lo que claramente influye en cómo se perciben las situaciones laborales ${ }^{29}$, 30 . Esta actitud positiva genera un estado de bienestar en el trabajador que le lleva a disfrutar de la tarea que realiza, así como de rol profesional, este sentimiento no suceden en experiencias como la adicción al trabajo donde el profesional pierde la capacidad para disfrutar con el trabajo ${ }^{31}$.

¿Cuáles son los factores que se relacionan con engagement? La mayoría de las investigaciones sobre engagement se han desarrollado de acuerdo con el modelo de demandas y recursos laborales que se ha definido anteriormente ${ }^{15}$ (Job DemandsResources model) donde se considera que el trabajador haría frente a las exigencias laborales a través de los recursos laborales. Desde este punto de vista, previos estudios han mostrado que los recursos laborales y personales facilitan engagement ${ }^{32,22,33}$. Los recursos laborales se refieren a los aspectos del trabajo que ayudan a manejar adecuadamente las demandas laborales, que permiten un óptimo funcionamiento, la consecución de objetivos y estimulan el crecimiento personal ${ }^{15}$. Estos recursos son particularmente importantes cuando el trabajo tiene un nivel alto de demandas y los trabajadores tienen que poner en práctica un repertorio importante de estrategias y habilidades $^{34}$. Algunos ejemplos de estos recursos laborales serían, la autonomía, el apoyo de los compañeros y supervisores, entornos laborales igualitarios y respetuosos, políticas de conciliación vida personal y laboral, etc. Los recursos personales, se refieren a variables vinculadas al propio trabajador que permiten una óptima adaptación con su entorno laboral, y que posibilitan una interacción positiva del trabajador con el medio del trabajo, generando emociones positivas y bienestar laboral.

\section{PERSONALIDAD POSITIVA Y RECURSOS PERSONALES}

Igualmente las investigaciones en salud laboral, han avanzado en cuanto a la clásica consideración exclusiva de los modelos tradicionales de la personalidad o en la focalización limitada en afectividad positiva y negativa para entender el bienestar laboral ${ }^{34,6,35,36}$. Actualmente, se ha ampliado la perspectiva de estudio sobre los recursos personales y las variables de personalidad positiva implicadas en la salud laboral y en el bienestar, haciendo hincapié en la importancia de las capacidades psicológicas y de resistencia del trabajador para el desarrollo óptimo laboral ${ }^{6,37,38,39}$.

Este planteamiento ofrece una aproximación a algunos aspectos positivos de los recursos personales, lo que ayuda a nuestra comprensión de los procesos de compromiso para conseguir la salud laboral y el bienestar. Desde este enfoque, los programas dentro de la organización destinados a conseguir un mayor bienestar, deberían también preocuparse por el fortalecimiento de los recursos personales, además de conllevar los respectivos cambios organizacionales. Por ejemplo, aprender a tener una visión optimista, el desarrollo de las competencias emocionales (la expresión verbal de las emociones y la empatía) y habilidades de personalidad resistente (es decir, sentirse con capacidad de control para modificar las condiciones adversas, y considerar que merece la pena invertir esfuerzo en cambiar aquello que genera malestar, así como, aprender a percibir desafíos en las situaciones cambiantes o nuevas). 
El enfoque centrado en el fortalecimiento de los recursos personales puede igualmente tener consecuencias positivas en la organización a través del desarrollo de las emociones positivas, la cooperación, la credibilidad, la creatividad, y la puesta en práctica de recursos que ayudan a cambiar la situaciones laborales negativas, y una mayor determinación hacia el bienestar ${ }^{40,41}$.

De las variables personales positivas las más vinculadas a la salud laboral han sido el optimismo ${ }^{42-44}$, la autoestima ${ }^{45-48}$, la personalidad resistente ${ }^{49-51}$, la competencia emocional $^{52,53}$ y la auto-eficacia ${ }^{54-56}$. No obstante, otras también influyentes han sido el sentido de la coherencia ${ }^{57}$ y el flujo. No hay duda de que estas variables positivas están relacionadas, pero no hay muchos estudios de varias de ellos simultáneamente. Desde esta perspectiva, podríamos hablar de dos modelos que tratan de tener en cuenta de manera conjunta estas variables: el modelo de Capital Psicológico de Luthans, Youssef, y Avolio $^{37}$ y el modelo de los Recursos Emocionales de Resistencia ${ }^{58,6}$.

\section{CAPITAL PSICOLÓGICO}

El constructo de Capital Psicológico (Pychological Capital; PsyCap) es considerado como un conjunto de recursos personales que recoge autoeficacia, atribuciones positivas como el optimismo y la esperanza que mantiene la ilusión hacia la consecución de objetivos $^{37}$. A nivel longitudinal, se ha observado que los trabajadores con variables de PsyCap explican un porcentaje significativo de la varianza en las puntuaciones de engagement, y que PsyCap ayuda a controlar las situaciones laborales conflictivas y los trabajadores muestran una mayor orientación al bienestar laboral ${ }^{59}$.

Desde este modelo se insiste en la importancia que tienen para las organizaciones de las relaciones laborales positivas entre la organización y los usuarios, y entre la organización y los trabajadores ${ }^{60}$. Para estos autores el verdadero motor de la salud laboral y el bienestar son los trabajadores y sus actitudes para generar entornos laborales constructivos y amigables, al referirse a los trabajadores, se piensa en todos los niveles profesionales, donde la dirección tiene que mostrar un compromiso para facilitar este bienestar laboral.

Las características específicas que se definen con PsyCap son las siguientes: (1) sentimiento personal de autoeficacia, esto quiere decir que el trabajador se siente capaz de cambiar las situaciones adversas, así como generar entornos de aprendizaje y desarrollo; (2) optimismo, expectativas positivas sobre el futuro y que las cosas se pueden cambiar para mejorar; (3) esperanza, perseverancia hacia las metas, así como cambiar los aspectos o metas según los objetivos personales y (4) resistencia, antes los problemas o adversidades, consiste en no abandonar las metas personales y trabajar en la consecución de los objetivos o reformularlos si fuera necesario ${ }^{37}$.

En definitiva, según proponen estos autores, estos recursos personales son la clave para conseguir el bienestar y el desempeño laboral sobresaliente, y pueden influir en la capacidad de aprendizaje por parte de los trabajadores.

No obstante, no hay muchos estudios actualmente que demuestren el impacto real de PsyCap, sólo los conducidos por Luthans, Avolio, Avey, y Norman ${ }^{61}$ donde los altos niveles de Psycap fueron relacionados con una mayor productividad y satisfacción laboral. Otros estudios de Luthans, Norman, Avolio y Avey ${ }^{62}$ han demostrado el efecto mediador de PsyCap entre clima organizacional y productividad. De manera adicional, en el estudio de diario de Xanthopoulou, Bakker, Heuven, Demerouti, y Schaufeli ${ }^{63}$ la productividad está relacionada con la autoeficacia (uno de los componentes de PsyCap) y engagement. Igualmente, se ha podido comprobar cómo los Recursos organizacionales (p.ej., autonomía, formación, clima laboral) están relacionados con cambios en la autoeficacia, autoestima y optimismo ${ }^{59}$. 


\section{RECURSOS EMOCIONALES DE RESISTENCIA}

Desde este modelo se insiste en la importancia del optimismo, la personalidad resistente y la competencia emocional para explicar el bienestar laboral, además de la importancia de las condiciones laborales positivas y el control de los factores de riesgo. El optimismo ha sido descrito como una actividad relacionada con la consecución de objetivos, expectativas positivas, y la autorregulación ${ }^{64}$. Desde esta perspectiva, el optimismo ayuda en la autorregulación y la planificación del futuro para gestionar los obstáculos y conseguir los objetivos. Igualmente el optimismo se relaciona con la confianza en que, a pesar de los obstáculos, el comportamiento persistente puede conseguir la realización de objetivos ${ }^{65}$.

Las personas optimistas se caracterizan por no vencerse ante la adversidad, y por tener una mayor flexibilidad cognitiva, esta visión positiva puede ayudar a tener un repertorio más amplio de solución de problemas. Son importantes los resultados de optimismo y una mayor vinculación con la realización personal y a un menor desgaste $^{42,43,44,66}$. Las personas optimistas planifican las situaciones difíciles ${ }^{67}$, son más persistentes $^{68}$ y tienen una perspectiva más flexible ante las situaciones de estrés ${ }^{69}$. Igualmente, algunas investigaciones relacionan el optimismo con una mayor satisfacción laboral y compromiso organizacional ${ }^{70-72}$.

La personalidad resistente ha sido entendida como una forma distintiva y activa de la comprensión de una persona con los demás, con los objetivos, con las situaciones cambiantes y con las situaciones laborales difíciles ${ }^{6,73,74}$. Este modelo de personalidad positiva se compone de tres variables ${ }^{75}$ : el compromiso se refiere a la participación en la vida y a la implicación en los aspectos relevantes para la vida de la persona, el control está relacionado con el reconocimiento de la influencia sobre los acontecimientos, especialmente en la situación adversa, y el desafío o reto es la orientación al cambio, a la búsqueda de situaciones y soluciones alternativas.

Actualmente, se han propuesto dos mecanismos para explicar el efecto de la personalidad resistente: una percepción más optimista de los acontecimientos ${ }^{76,77}$ y el uso de determinadas estrategias de afrontamiento más centrado en la búsqueda de solución al problema y la reformulación de los acontecimientos y sus consecuencias ${ }^{78,79}$. Según Ford-Gilboe y Cohen $^{80}$, la forma de afrontamiento de los individuos con personalidad resistente consiste en la transformación de los acontecimientos estresantes directamente o si esto no fuera posible, cambiar la percepción de las situaciones. Esta forma de realización puede movilizar distintos recursos para el perfeccionamiento y mejora de las condiciones laborales. Otra acción complementaria es la práctica de hábitos saludables (p.ej., utilizar el ejercicio físico en situaciones de estrés), una consecuencia de las creencias sobre la responsabilidad de la salud y la felicidad, también en las organizaciones.

La personalidad resistente se asociación de manera positiva con la efectividad organizacional $^{80-83}$ y negativamente con el burnout o desgaste profesional ${ }^{53,66}$.

La competencia emocional se refiere a las habilidades necesarias en las interacciones socio-emocionales ${ }^{84}$ y en el respeto a los compañeros y usuarios en las relaciones del día a día. En el contexto de trabajo, la competencia emocional implica un manejo de las emociones, teniendo en cuenta los valores morales y éticos, donde la cooperación y la ayuda mutua forman parte del trabajo en equipo de manera natural, y los valores profesionales son valorados.

De acuerdo con Saarni, la competencia emocional tiene consecuencias muy apreciables en el comportamiento: la habilidad para manejar las propias emociones con las estrategias de afrontamiento adaptativas, la sensación de bienestar subjetivo facilitado por la existencia de valores morales y éticos, y la fortaleza ante situaciones adversas. Estas consecuencias han sido confirmadas en contextos personales, sociales, y en el lugar de trabajo $^{85,86,87,88,89,6,90,91}$. 


\section{BUENAS PRÁCTICAS ORGANIZACIONALES E INDIVIDUALES QUE FOMENTAN LA SALUD LABORAL Y EL BIENESTAR}

En definitiva, de acuerdo a estos modelos planteados se proponen una serie de buenas prácticas que tienen por objetivo, orientarse a la salud y al bienestar laboral, a través de la interacción y el equilibrio de cambios positivos organizacionales y en el desarrollo de los recursos saludables y positivos por parte del trabajador. Las intervenciones centradas en la interacción del contexto laboral y el individuo entienden la salud laboral y el bienestar como fruto de la relación del trabajador y el medio laboral. Entre otros factores, se trataría de mejorar las condiciones laborales, los recursos del trabajador y la forma de afrontamiento ante las situaciones de malestar laboral, todo ello de una manera integrada.

Entre los principales factores psicosociales que deben seguir este planteamiento, es decisiva la cultura organizacional saludable; así, las organizaciones óptimas, son aquellas que comparten valores y comportamientos éticos. Desde este punto de vista, las organizaciones no son simples estructuras severas de funcionamiento, sino que las organizaciones tienen su singularidad y unos valores que las identifican. Cuando la organización es saludable transmite a sus trabajadores un ambiente sano que se caracteriza por valores éticos, emociones positivas como el compañerismo, un estilo de trabajar cooperando y con profesionalidad, que fomenta las relaciones sociales y el apoyo mutuo.

Desde esta perspectiva, la cultura puede fomentar los aspectos positivos en los trabajadores como el apoyo social, el respeto por la singularidad y diversidad, la autonomía, la ética, etc. Sin embargo, existen igualmente culturas organizacionales tóxicas que pueden dar lugar a disfunciones organizacionales o ser principio de problemas o conductas hostiles y de discriminación para los trabajadores que puede fomentar patologías laborales como el acoso psicológico, sexual, etc.

De manera contraria, la cultura de respeto y saludable, contribuye a generar un clima laboral positivo, que consiste en la satisfacción con las relaciones interpersonales. La importancia de las relaciones emocionales, en la medida que exista un entorno amistoso de trabajo, las relaciones se establecerán de una forma más segura y positiva. Las organizaciones con un clima negativo de trabajo fomentan emociones hostiles y entornos poco amistosos, donde se pueden favorecer situaciones de estrés y conductas violentas.

Evitar el estrés de rol, otra disfunción importante y frecuente en las organizaciones que viene determinada por las situaciones de conflicto y ambigüedad de rol. Estas características negativas generan situaciones que se relacionan con el estrés y las sobrecarga lo que se afecta a la probabilidad de sufrir en mayor medida riesgos psicosociales (p.ej., desgaste profesional, conflictos interpersonales, etc.). La clarificación de roles permite una sensación de control por parte del trabajador, así como evitar situaciones de ambigüedad derivadas por la escasa clarificación de funciones. Igualmente, es importante la posibilidad de tener unas condiciones de trabajo que eviten la sobrecarga con una distribución más equitativa del trabajo y en relación a los recursos del trabajador.

El liderazgo, supone un manejo adecuado de las habilidades de comunicación, motivación, resolución de conflictos y desarrollo de equipos, pero tales funciones no resultan efectivas sin una buena interacción con los miembros del equipo y los objetivos éticos de las organizaciones. Un liderazgo ético, eficaz y atento a las normas y valores positivos de la organización puede evitar problemas graves en las organizaciones, como las situaciones de agresión laboral (p.ej., acoso psicológico y sexual). Cuando en la organización hay un liderazgo flexible y eficaz, la organización es más competente para aplicar los valores éticos y de respeto mutuo, así como orientarse al bienestar y la felicidad.

La organización está compuesta por personas, desde este punto de vista el apoyo social por parte de la organización, es el elemento más relevante para buscar soluciones en las situaciones problemáticas. Si los trabajadores cuentan con apoyo social por parte 
de la organización, tienen una menor probabilidad de experimentar patologías laborales. Ante un problema laboral, es fundamental para el trabajador que pueda contar con el reconocimiento y la ayuda mutua. Así, se produce una estrategia de solución de problemas compartidos, donde no se generan estrategias de evitación que pueden llevar al desarrollo de espirales negativas o activar situaciones de violencia o agresión. De la misma manera, el contacto social favorece las relaciones de amistad y reduce los sentimientos de soledad. La pertenencia al grupo hace posible el establecimiento y consecución de metas que no podrían ser conseguidas por un solo individuo. Desde esta perspectiva una organización que fomenta el apoyo social, es fuente de satisfacción, se produce una comunicación fluida y genera sentimientos de pertenencia, socialización y que, además, pueden ser factores de protección de situaciones estresantes.

La participación es un elemento muy importante de mejora. Contribuye a la formación y al crecimiento personal de quienes participan, puesto que implica el uso de técnicas de resolución de problemas, análisis de las situaciones, buscar alternativas, trabajar en equipo, mejorar la comunicación, etc. La participación también está muy vinculada al desarrollo profesional en términos de competencias, hasta qué punto la organización posibilita la puesta en práctica de las habilidades o competencias del trabajador. Un trabajo que posibilita el desarrollo personal y profesional debe permitir al trabajador sentir que su trabajo sirve para algo, que tiene una utilidad en el conjunto del proceso en el que se desarrolla y también para la sociedad en general. Además debe ofrecerle la posibilidad de aplicar y desarrollar sus conocimientos y capacidades, así como descubrir nuevas experiencias y posibilidades.

\section{PRACTICAR LOS RECURSOS EMOCIONALES DE RESISTENCIA}

El entrenar los recursos personales de resistencia se plantea dentro de un contexto laboral, donde los recursos de los individuos juegan un papel fundamental, por lo que se presta especial atención a las habilidades positivas personales que estarían relacionadas con el bienestar en las organizaciones. Tal y como se ha comentado, los recursos emocionales de resistencia delimitan un conjunto de variables relevantes para conocer y predecir las fuentes de resistencia a las patologías psicosociales. Así, incluye la personalidad resistente, el optimismo y la competencia emocional.

El desarrollo de este constructo parte de una concepción de la personalidad como un sistema de procesos cognitivos, emocionales y conductuales que interactúan entre sí, cambiándose consecutivamente, pero que también mantienen una relativa independencia ${ }^{92}$. Desde este modelo se han seleccionado variables que tienen componentes emocionales y motivacionales, relacionados con estados de ánimo positivos que potencian los procesos de salud y bienestar del individuo en el trabajo.

El profesional pueden aprender a manejar y regular sus emociones, y conseguir entender las emociones de los demás (p. ej, manejar las situaciones difíciles con los usuarios, los compañeros, etc.), aspectos estos que tienen que ver con el manejo de pensamientos, imágenes, emociones, etc., y que implican una comprensión de la competencia emocional y de los recursos personales para resolver las dificultades laborales. Por ello, los profesionales con un repertorio amplio de estrategias de competencia emocional resolverán un mayor número de situaciones problemáticas.

Especialmente, estas habilidades son imperiosas en el caso de los trabajadores de tipo asistencial que requieren el manejo de las emociones como una parte importante de su trabajo, además de responder a las exigencias físicas y mentales de las características propias de sus trabajos. Estos profesionales tienen que tener competencias personales que les permita planificar, manejar y regular sus necesidades para expresar las emociones deseadas durante las transacciones interpersonales en el lugar del trabajo, y además conseguir alcanzar los objetivos planteados organizacionales. 
Finalmente, las habilidades emocionales son relevantes y tienen importantes aplicaciones en el trabajo ${ }^{93}$ específicamente en la generación de un clima laboral positivo ${ }^{94}$ lo que a su vez se relaciona con la productividad y la calidad de vida de los trabajadores ${ }^{95,82,96}$. De esta perspectiva, la expresión de las emociones, las emociones positivas y la sensibilidad hacia las emociones de los otros puede fomentar el compromiso, la eficacia y la realización personal.

Educar y fomentar los procesos emocionales de resistencia implica también trabajar la personalidad resistente del individuo para fomentar una actitud ante la vida de compromiso, control y reto. Esta percepción sobre el mundo, los otros y sí mismo, implica una serie de estrategias o habilidades para resolver las distintas situaciones de la vida, el estrés y modificar el estado de ánimo negativo. Estas actitudes incluyen diferentes estrategias de afrontamiento, y de interacción social, así como prácticas de autocuidado (la relajación, la alimentación saludable, el ejercicio físico, etc..). La personalidad resistente tiene que ver con aprender a evaluar las situaciones cambiantes como oportunidades, como retos y no como peligros.

Las personas con estas características se implican en el trabajo y con las personas. Igualmente, la percepción de control sobre la vida, ayuda a manejar de una manera más adecuada los estresores y a tomar la decisiones más positivas para el individuo ${ }^{75}$. Las personas con estas características de resistencia no evitan las situaciones o aguantan sin más ante los acontecimientos negativos, son capaces de tomar una decisión en su propio beneficio y en el de la organización, así como manifestar las situaciones injustas o que generan malestar. No son personas pasivas, que resisten sin crítica, son todo lo contrario, personas que están dispuestas a cambiar las situaciones negativas y que tratan de orientarse a la salud y el bienestar, modificando los aspectos que se pueden alejar de sus objetivos en este sentido.

De igual manera, otro elemento importante consiste en fomentar el optimismo, sobre todo la expectativa generalizada de que las cosas se resolverán de forma positiva ${ }^{97}$. Desde esta perspectiva los profesionales aprenden que el pensamiento influirá de manera importante en las metas personales, en la definición de los valores deseados y en los proceso de autorregulación ${ }^{98}$. El aprendizaje del optimismo implica un proceso o mecanismo de autorregulación que las personas utilizan para conseguir las metas, descubrir los impedimentos que pueden encontrarse en el camino, y elegir la estrategia más adecuada.

Una actitud optimista ante la vida aumenta la probabilidad de mantener un continuo esfuerzo por conseguir los objetivos, la persistencia y la determinación de la acción, mientras que las personas con actitudes pesimistas abandonan prontamente. Desde esta perspectiva, los profesionales aprenden que una disposición optimista está relacionada con los resultados y con las estrategias de afrontamiento activas y efectivas ${ }^{64}$. El planteamiento que aquí se trata de seguir es la consecución de los objetivos que se marca la persona y la organización.

El optimismo eficaz es aquel que es capaz de comprender la situación de una manera crítica, así con la demostración de la capacidad de expresar abiertamente los elementos que necesitan cambios y que no se corresponden con los valores éticos y de respeto de la organización, a la par que con los objetivos personales. Las personas optimistas en el medio laboral, mantienen la esperanza, pero son críticas con las situaciones que tienen que modificarse y deben solucionarse, y trabajan para realizar cambios e intentan buscar soluciones creativas.

Para desarrollar la competencia emocional es importante trabajar una serie de capacidades que tienen que ver con el conocimiento de las propias emociones, las habilidades para diferenciar y entender las emociones de otros, las habilidad para usar un vocabulario amplio y variado de expresión de las emociones, la capacidad para empatizar, las habilidades para diferenciar entre la expresión emocional interna y externa, el conocimiento de la comunicación emocional dentro de las diferentes relaciones sociales 
y la capacidad de autoeficacia emocional ${ }^{96}$. La competencia emocional, aplicada al trabajo, consiste en trasladar este contenido al mundo laboral.

La competencia emocional en el lugar de trabajo hace referencia a dos niveles distintos, pero conectados entre sí, el nivel intrapersonal y el nivel interpersonal. El nivel intrapersonal, implica cómo desarrollar las competencias emocionales y cómo usarlas personalmente. En cuanto al nivel interpersonal, todas estas competencias serán extendidas al ámbito social, a las relaciones con los demás, para ser más efectivos en las relaciones con los otros.

La competencia emocional de los trabajadores ayuda en la creación de una cultura de respeto, ética y de atención entre los trabajadores y hacia los usuarios o clientes, un sistema directivo respetuoso y atento a las formas, una mayor adaptación a las exigencias laborales, un sistema de comunicación eficaz y flexible, una gestión más eficaz de la organización, una aceptación de la expresión de las emociones negativas ${ }^{82,99}$.

En definitiva, estos recursos tendrán una relación directa con la valoración positiva del trabajador lo que se relaciona con una mayor efectividad en las relaciones interpersonales ${ }^{100}$. Igualmente, esta visión positiva de sí mismo se ha considerado como un importante recurso frente a los estresores laborales ${ }^{101}$. Maslach ${ }^{102}$ reconoce la importancia de los sentimientos positivos en el contexto laboral y en relación al desgaste profesional su implicación en los procesos de autoevaluación y autoconceptualización.

Los estudios realizados ponen de manifiesto que cuando los trabajadores se sienten con menos recursos frente al estrés hay una mayor vulnerabilidad frente a los riesgos psicosociales. Cuando un trabajador tiene sentimientos negativos sobre sí mismo, puede mostrar incompetencia en las relaciones interpersonales (pacientes/usuarios y compañeros de trabajo) los que puede generar dificultades en el contexto laboral. Sin embargo, la valoración positiva del trabajador cuando se percibe con recursos, se relaciona con la eficacia $^{103}$. Desde la perspectiva de aumento de los recurso, es importante fomentar los aspectos valorables de los profesionales, los logros personales y profesionales, trabajar una perspectiva positiva sobre sí mismo, el aumento de los niveles de competencia y el aumento del valor social del profesional, así como una cultura de respeto y ética compartida.

Concluyentemente, además de los cambios organizaciones propuestos, trabajar las variables positivas de los trabajadores, genera un aumento de los recursos personales, lo que se relaciona con una mayor autoestima, mayor frecuencia de emociones positivas y una mayor competencia emocional ${ }^{6,104,105}$.

Todas estas variables definidas se trabajan de manera vinculada para desarrollar los recursos emocionales de resistencia que actuarían cómo elementos protectores de las patologías psicosociales y que aumentan la probabilidad de que los trabajadores desarrollen el bienestar en las organizaciones. Los recursos emocionales de resistencia tienen un marcado carácter saludable, estarían vinculados a puntuaciones más bajas de estrés, a estados emocionales positivos, al uso de estrategias de afrontamiento adaptativas, a puntuaciones altas en engagement y autoeficacia, y a un mayor bienestar subjetivo ${ }^{6}$.

En conclusión, los modelos aquí esbozados sugieren la importancia de la salud laboral y el bienestar para proporcionar resultados favorables en el trabajo. Los beneficios de estos planteamientos implican efectos deseables con respeto a la actividad en la tarea, la persistencia, el funcionamiento cognitivo y la creatividad, así como en la calidad del servicio prestado y en las relaciones positivas interpersonales, donde se contribuye a desarrollar y proteger el respeto mutuo.

Las personas que trabajan en organizaciones saludables, se benefician de las emociones positivas mutuas y de las acciones positivas de los otros al verse influidos por ellos. Por último, se producen comportamientos éticos, altruistas y de cooperación con los otros, así como un entorno laboral comprometido con el respeto. Todo ello derivaría en unos resultados favorables en el lugar del trabajo. 


\section{REFERENCIAS BIBLIOGRÁFICAS}

1. Seligman M, Csikszentmihalyi M. Positive Psychology: An introduction. Am Psychol 2000; 55: 5-14.

2. Bakker AB, Rodriguez-Muñoz A. Positive Occupational Health Psychology: An introduction. Psicothema; In press.

3. Fredrickson BL, Joiner T. Positive emotions trigger upward spirals toward emotional well-being. Psychol Sci 2002; 13 (2): 172-175.

4. Erez A, Isen AM. The influence of positive affect on the components of expectancy motivation. J Appl Psychol 2002; 87: 1055-1067.

5. Lim S, Cortina LM, Magley VJ. Personal and workgroup incivility: Impact on work and health outcomes. J Appl Psychol 2008; 93 (1): 95-107.

6. Garrosa E, Moreno-Jiménez B, Rodríguez-Muñoz A, Rodríguez-Carvajal R. Role stress and persona resources in nursing: A cross-sectional study of burnout and engagement. Int J Nurs Stud 2011; 48: 479-489.

7. Fredrickson BL, Branigan C. Positive emotions broaden the scope of attention and thought-action repertoires. Cognition and Emotion 2005; 19 (3): 313-332.

8. Rowe G, Hirsh JB, Anderson AK. Positive affect increases the breadth of attentional selection Proceedings of the National Academy of Sciences USA 2007; 104 (1): 383-388.

9. Isen AM, Daubman KA, Nowicki GP. Positive affect facilitates creative problem solving. J Pers Soc Psychol 1987; 52 (6): 1122-1131.

10. Kahn BE, Isen AM. The influence of positive affect on variety seeking among safe, enjoyable products. Journal of Consumer Research 1993; 20 (2): 257-270.

11. Raghunathan R, Trope Y. Walking the tightrope between feeling good and being accurate: Mood as a resource in processing persuasive messages. J Pers Soc Psychol 2002; 83 (3): 510-525.

12. Waugh CE, Fredrickson BL. Nice to know you: Positive emotions, selfother overlap, and complex understanding in the formation of a new relationship. The Journal of Positive Psychology 2006; 1 (2): 93-106.

13. Dunn JR, Schweitzer ME. Feeling and believing: The influence of emotion on trust. J Pers Soc Psychol 2005; 88 (5): 736-748.

14. Peterson U, Demerouti E, Bergström G, Åsberg M, Nygren Å. Work characteristics and sickness absence in burnout and non burnout groups: A study of Swedish health care workers. International Journal of Stress Management 2008; 15 (2): 153-172.

15. Bakker AB, Demerouti E. The Job Demands-Resources model: State of the art. Journal of Managerial Psychology 2007; 22: 309-328.

16. Bakker AB, Demerouti E, Euwema MC. Job resources buffer the impact of job demands on burnout. J Occup Health Psychol 2005; 10: 170-180.

17. Demerouti E, Bakker AB, Nachreiner F, Schaufeli WB. A model of burnout and life satisfaction amongst nurses. J Adv Nurs 2001; 32: 454-464.

18. Schaufeli WB, Bakker AB. Job demands, job resources and their relationship with burnout and engagement: A multi-sample study. Journal of Organizational Behavior 2004; 25: 293-315.

19. Bakker AB, Hakanen JJ, Demerouti E, Xanthopoulou D 2007. Job resources boost work engagement, particularly when job demands are high. Journal of Educational Psychology 2007; 99: 274-284.

20. Demerouti E, Bakker AB, de Jonge J, Janssen PPM, Schaufeli WB. Burnout and engagement at work as function of demands and control. Scand J Work Environ Health 2001; 27: 279-286.

21. Lee RT, Ashforth BE. A meta-analytic examination of the correlates of the three dimensions of job burnout. J Appl Psychol 1996; 81: 123-133.

22. Bakker AB, Demerouti E, Ten Brummelhuis LL. Work engagement, performance, and active learning: The role of conscientiousness. Journal of Vocational Behavior; 2011 - in press.

23. Bakker AB, Bal PM. Weekly work engagement and performance: A study among starting teachers. Journal of Occupational and Organizational Psychology 2010; 83: 189-206.

24. Halbesleben JRB, Wheeler AR. The relative roles of engagement and embeddedness in predicting job performance and intention to leave. Work \& Stress 2008; 22: 242-256.

25. Salanova M, Agut S, Peiró JM. Linking organizational resources and work engagement to employee performance and customer loyalty: The mediation of service climate. J Appl Psychol 2005; 90: 1217-1227. 
26. Demerouti E, Cropanzano R. From thought to action: Employee work engagement and job performance. In: Bakker AB, Leiter MP, Eds. Work engagement: A handbook of essential theory and research. New York: Psychology Press; 2010. p. 147-163.

27. Schaufeli WB, Bakker AB. Defining and measuring work engagement: Bringing clarity to the concept. In: Bakker AB, Leiter MP, Eds. Work engagement: A handbook of essential theory and research. New York: Psychology Press; 2010. p. 10-24.

28. Schaufeli WB, Salanova M, González-Romá V, Bakker AB. The measurement of engagement and burnout: a confirmative analytic approach. Journal of Happiness Studies 2002; 3: 71-92.

29. Bakker. Building engagement in the workplace. In: Cooper C, Burke R, Eds. The peak performing organization. London: Routledge; 2009. p. 50-72.

30. Schaufeli WB, Taris TW, Le Blanc P, Peeters M, Bakker AB, De Jonge J. Maakt arbeid gezond? Op zoek naar de bevlogen werknemer [Does work make happy? In search of the engaged worker]. De Psycholoog 2001; 36: 422-428.

31. Gorgievski MJ, Bakker AB, Schaufeli WB. Work Engagement and Workaholism: Comparing the SelfEmployed and Salaried Employees. Journal of Positive Psychology 2010; 5 (1): 83-96.

32. Bakker AB, Demerouti E. Towards a model of work engagement. Career Development International 2008; 13: 209-223.

33. Bakker AB, Hakanen JJ, Demerouti E, Xanthopoulou D. Job resources boost work engagement, particularly when job demands are high. Journal of Educational Psychology 2007; 99: 274-284.

33. Halbesleben, J. R. B., \& Wheeler, A. R. (2008). The relative roles of engagement and embeddedness in predicting job performance and intention to leave. Work \& Stress, 22, 242-256.

34. Cooper CL. Introduction: a discussion about the role of negative affectivity in job stress research. Journal of Organizational Behavior 2000; 21: 77-78.

35. Payne RL. Comments on "Why negative affectivity should not be controlled in job stress research: Don't throw out the baby with the bath water". Journal of Organizational Behavior 2000; 21: 97-100.

36. Spector PE, Zapf D, Chen PY, Frese M. Why negative affectivity should not be controlled in job stress research: don't throw out the baby with the bathwater. Journal of Organizational Behavior 2000; 21: 79-96.

37. Luthans F, Youssef CM, Avolio BJ. Psychological capital: Developing the human competitive edge. Oxford, UK: Oxford University Press; 2007

38. Varvel SJ, He Y, Shannon JK, Tager D, Bledman RA, Chaichanasakul A, Mendoza MM, Mallinckrodt B. Multidimensional threshold effects of social support in firefighters: is more support invariably better? Journal of Counseling Psychology 2007; 54 (4): 458-465.

39. Zellars KL, Hochwarter WA, Perrewé PL, Hoffman N, Ford EW. Experiencing job-burnout: the roles of positive and negative traits and states. Journal of Applied Social Psychology 2004; 34: 887-911.

40. Maddi SR. The story of hardiness: twenty years of theorizing, research, and practice. Consulting Psychological Journal 2002; 54: 173-185.

41. Maddi SR, Khoshaba DM, Pammenter A. The hardy organization: Success by turning change to advantage. Consulting Psychological Journal: Practice and Research 1999; 1: 117-124.

42. Chang EC, Rand KL, Strunk DR. Optimism and risk for job burnout among working college students stress as a mediator. Personality and Individual Differences 2000; 29: 255-263.

43. Grau A, Suñer R, García M. Desgaste profesional en el personal sanitario y su relación con los factores personales y ambientales.(-Burnout in health care professionals and its relation with personal and environment factors). Gaceta Sanitaria 2005; 19: 463-469.

44. Riolli L, Savicki V. Information system organizational resilience. Omega 2003; 31: 227-233.

45. Browning L, Ryan CS, Greenberg MS, Rolniak S. Effects of cognitive adaptation on the expectationburnout relationship among nurses. J Behav Med 2006; 29: 139-150.

46. Janssen J. June. Self-enforcement of joint implementation and clean development mechanism contracts In: Paper Presented at the First World Conference of Environmental and Resource Economists, Venice; 1999.

47. Mäkikangas A, Kinnunen U. Psychosocial work stressors and wellbeing: Self-esteem and optimism as moderators in a one-year longitudinal sample. Personality and Individual Differences 2003; 35: 537-557.

48. Pruessner JC, Hellhammer SDH, Kirschbaum C. Low self-esteem induced failure and the adrenocortical stress response. Personality and Individual Differences 1999; 27: 477-489.

49. Chang WC. The lived experience of role transition of being advanced practice nurses in collaboration. Unpublished thesis. National Taipei College of Nursing, Taipei, Taiwan; 2003. 
50. Demerouti E, Bakker AB, Voydanoff P. Does home life interfere with or facilitate performance? European Journal of Work and Organizational Psychology 2010; 19: 128-149.

51. Michielsen HJ, Willemsen TM, Croon M, De Vries J, Van Heck GL. Determinants of general fatigue and emotional exhaustion: a prospective study. Psychology and Health 2004; 19: 223-235.

52. Cherniss C. Emotional Intelligence: What it is and Why it Matters. Issues in Emotional Intelligence. [Electronic Version]. Volume not included 2000; 1-14.

53. Garrosa E, Moreno-Jiménez B, Liang Y, González JL. The relationship between socio-demographic variables, job stressors, burnout, and hardy personality in nurses: an exploratory study. Int $\mathrm{J}$ Nurs Stud 2008; 45 (3): 418-427.

54. Cherniss C. The role of professional self-efficacy in the etiology of burnout. In: Schaufeli WB, Maslach C, Marek T, Eds. Professional Burnout: Recent Developments in Theory and Research. London: Taylor \& Francis; 1993. p. 135-149.

55. Salanova M, Grau R, Martínez I. Job demands and coping behaviour: the moderating role of professional self-efficacy. Psychology in Spain 2005; 10: 1-7.

56. Xanthopoulou D, Bakker AB, Demerouti E, Schaufeli WB. The role of personal resources in the job demands-resources model. International Journal of Stress Management 2007; 14: 121-141.

57. Moreno-Jiménez B, Garrosa E, González JL. El desgaste professional de enfermería: Desarrollo y validación factorial del CDPE (Professional nurses' burnout. Development and factor validation of the CDPE). Archivos prevención de riesgos laborales 2000; 3: 18-28.

58. Garrosa E. 2003. Los procesos emocionales de resistencia: un afrontamiento adaptativo ante el desgaste profesional. Estudio en una muestra de enfermería. Tesis doctoral no publicada. Facultad de psicología. Universidad Autónoma de Madrid.

59. Xanthopoulou D, Bakker AB, Demerouti E, Schaufeli WB. Work engagement and financial returns: A diary study on the role of job and personal resources. Journal of Occupational and Organizational Psychology 2009; 82: 183-200.

60. Larson M, Luthans F. Potential added value of psychological capital in predicting work attitudes. Journal of Leadership and Organizational Studies 2006; 13: 45-62.

61. Luthans F, Avolio BJ, Avey JB, Norman SM. Psychological capital: Measurement and relationship with performance and satisfaction. Personnel Psychology 2007; 60: 541-572

62. Luthans F, Norman SM, Avolio BJ, Avey JB. The mediating role of psychological capital in the supportive organizational climate-employee performance relationship. Journal of Organizational Behavior 2008; 29: 219-238.

63. Xanthopoulou D, Bakker AB, Heuven E, Demerouti E, Schaufeli WB. Working in the sky: A diary study on work engagement among flight attendants. Journal of Occupational Health Psychology 2008; 13: 345-356.

64. Scheier MF, Carver CS. Optimism, coping and health: assessment and implications of generalized outcome expectancies. Health Psychology 1985; 4: 219-247.

65. Lebsack RR, Lebsack S, Luthans KW. Positivity in the workplace: the impact of nurses' optimism on their rated performance. Midwest Academy of Management. 2004

66. Moreno-Jiménez B, Garrosa E, Corzo S, Boada M. Personalidad Resistente y Capital Psicológico: las variables personales positivas y los procesos de agotamiento y vigor. Psicothema; en prensa.

67. Strack S, Carver CS, Blaney PH. Predicting successful completion of an aftercare program following treatment for alcoholism: the role of dispositional optimism. Journal of Personality and Social Psychology 1987; 53: 579-584.

68. Seligman MEP. Learned Optimism. New York: Simon \& Schuster; 1998

69. Carver CS, Gaines JG. Optimism, pessimism, and postpartum depression. Cognitive Therapy and Research 1987; 11: 449-462.

70. Lum L, Kervin J, Clark K, Reid F, Sirola W. Explaining nursing turnover intent: job satisfaction, pay satisfaction, or organizational commitment? Journal of Organizational Behavior 1998; 19: 305-320.

71. Segerstrom SC. Optimism and resources: effects on each other and on health over 10 years. Journal of Research in Personality 2007; 41: 772-786.

72. Wanberg CR, Banas JT. Predictors and outcomes of openness to changes in a reorganizing workplace. Journal of Applied Psychology 2000; 85: 132-143

73. Maddi SR, Harvey RH, Khoshaba DM, Lu JL, Persico M, Brow M. The personality construct of hardiness. III: relationships with repression, innovativeness, authoritarianism, and performance. Journal of Personality 2006; 74: 575-598. 
74. Kobassa-Ouellette SC, Di Placido J. Personality's role in the protection and enhancement of health: where the research has been, where it is stuck, how it might move. In: Baum E, Revenson TA, Singer JE, Eds. Handbook of Health Psychology. NJ: Erlbaum, Mahwah; 2001. p. 175-193.

75. Kobasa SC. The hardy personality: toward a social psychology of stress and health. In: Sanders GS, Sals J, Eds. Social Psychology of Health and Illness. NJ: Erlbaum, Hillsdale; 1982. p. 3-32.

76. Alfred KD, Smith TW. The hardy personality: cognitive and physiological responses to evaluative threat. Journal Personality and Social Psychology 1989; 56: 257-266.

77. Wiebe DJ. Hardiness and stress moderation: a test of proposed mechanisms. Journal Personality and Social Psychology 1991; 60: 89-99.

78. Westman M. The relationships between stress and performance. The moderating effects of hardiness. Human Performance 1990; 3: 141-155.

79. Williams PG, Wiebe DJ, Smith TW. Coping processes as mediators of the relationship between hardiness and health. Journal Behaviour Medicine 1992; 15: 237-255.

80. Ford-Gilboe MC, Cohen JA. Hardiness: a model of commitment, challenge and control. In: Rice H, Ed Handbook of Stress, Coping and Health. Implications for Nursing Research, Theory and Practice. Thousand Oaks, CA: Sage; 2000. p. 425-436.

81. Moreno-Jiménez B, Morett NI, Rodríguez-Muñoz A, Morante ME. La personalidad resistente como variable moduladora del síndrome de burnout en una muestra de bomberos.(Hardy personality as amoderating variable of burnout in a sample of firemen). Psicothema 2006; 18: 413-418.

82. Garrosa E. Los procesos emocionales de resistencia: un afrontamiento adaptativo ante el desgaste professional. (The Emotional Processes of Resistance: Adaptive Coping to Professional Burnout). Madrid: Ediciones Universidad Autónoma; 2006.

83. Pollock SE. Human responses to chronic illness: physiological and psychosocial adaptation. Nursing Research 1986; 35: 90-95.

84. Saarni C. The Development of Emotional Competence. New York: Guilford Press; 1999.

85. Asher S, Rose A. Promoting children's social-emotional adjustment with peers. In: Salovey P, Sluyter D, Eds. Emotional Development and Emotional. New York: Basic Books; 1997. p. 196-224.

86. Compas B, Worsham N, Ey S. Conceptual and developmental issues in children's coping with stress. In Greca AL, Siegal L, Wallander J, Walker C, Eds. Stress and Coping in Child Health. New York: Guilford Press; 1992. p. 7-24.

87. Crick N, Dodge K. A review of social-information processing mechanisms in children's social adjustment Psychological Bulletin 1994; 115: 74-101.

88. Denham SA. Emotional Development in Young Children. New York: Guilford; 1999

89. Garber J, Braafladt N, Zeman J. The regulation of sad affect: an information processing perspective. In: Garber J, Dodge K, Eds. The Development of Emotion Regulation and Dysregulation. New York: Cambridge University Press; 1991. p. 208-240.

90. Parke RD, Cassidy J, Burks V, Carson J, Boyum L. Familial contribution to peer competence among children: the role of interactive and affective processes. In: Parke R, Ladd G, Eds. Familypeer Relationships: Modes of Linkage. NJ: Erlbaum, Hillsdale; 1992. p. 107-134.

91. Wolchik SA, Sandler IN. Handbook of Children's Coping: Linking Theory and Intervention. New York: Plenum Press; 1997.

92. Moreno Jiménez B. Psicología de la personalidad. Procesos. Madrid: Thomson Paraninfo; 2007.

93. Boyatzis RE. The Competent Manager. NY: Wiley; 1982.

94. Kelner S, Rivers C, O’Connell K. Managerial style as a behavioural predictor climate. Boston: McBer; 1994

95. Cherniss C, Goleman D. The emotionally intelligent workplace. San Francisco, CA: Jossey-Bass; 2001.

96. Saarni C. Emotional competence. A developmental perspective. In: Bar-On R, Parkes JDA, Eds. The Handbook of Emotional Intelligence. San Francisco: Jossey-Bass; 2000. p. 68-91.

97. Carver CS, Scheier MF. Origins and functions of positive and negative affect: A control-process view. Psychological Review 1990; 97: 19-35.

98. Sundstrom E, DeMeuse KP, Futrell D. Work teams: applications and effectiveness. Am Psychol 1990; 45: 120-133.

99. Moreno-Jiménez B, Garrosa E, Galindo C, San Julián L, Rodríguez R, Morante ME, Losada M. Manual sobre acoso psicológico: Transportes y comunicaciones de la Comunidad de Madrid. Madrid: UGT; 2002. 
100. Ross L. The intuitive psychologist and his shortcomings: Distortions in the attribution process. In: Berkowitz L, Ed. Advances in experimental social psychology. New York: Academic Press; 1977. p. 173-240.

101. Hobfoll SE, Freedy J. Conservation of resources: A general stress theory applied to burnout. In: Schaufeli WB, Maslach C, Marek T, Editors. Professional burnout: Recent developments in theory and research. Washington, DC: Taylor \& Francis; 1993.

102. Maslach C. Burnout: A multi-dimensional perspective. In: Schaufeli WB, Maslach C, Marek T, Editors. Professional burnout: Recent developments in theory and research. Washington, DC: Taylor \& Francis; 1993.

103. Brockner J. Self-esteem at work: Research, theory and practice. Lexington, MA: Lexington Books; 1988.

104. Moreno-Jiménez B, Rodríguez-Muñoz A, Garrosa Hernández E, Morante Benadero ME. Antecedentes organizacionales del acoso psicológico en el trabajo: un estudio exploratorio. Psicothema 2005; 17 (4): 648-653.

105. Zapf D, Einarsen S, Hoel H, Vartia M. Empirical findings on bullying in the workplace. In: Einarsen S, Hoel H, Zapf D, Cooper CL, Eds. Bullying and emotional abuse in the workplace: International perspectives in research and practice. London: Taylor \& Francis; 2003.

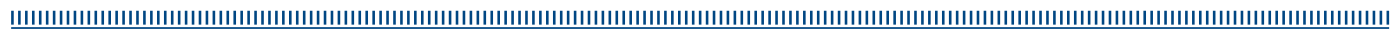

